\title{
Design of a Functional H Filter for Linear Singular Systems With an Additional Unknown Input, Bounded Disturbance, and Variable Time Delay
}

\author{
Hamzaoui Fatma, University of Monastir, Tunisia \& National Engineering School of Monastir, Tunisia \\ Khadhraoui Malek, National Engineering School of Monastir, Tunisia
}

Messaoud Hassani, National Engineering School of Monastir, Tunisia

\begin{abstract}
This paper deals with a new method for the design of a functional filter for linear singular systems with bounded disturbance and variable time delay in the state and the known input vectors. Moreover, a disturbance unknown inputs is considered. The main objective of such design is the estimation of functional state vector, and a part of the disturbance unknown inputs for fault detection. The proposed design is based on Lyapunov-Krasovskii stability theory and, in order to minimize the disturbance effect, the designed filter satisfies a $H_{-} \infty$ criterion. Numerical simulation is used to illustrate the proposed design and the results were successful.
\end{abstract}

\section{KEYWORDS}

Bounded Disturbance, Functional Filter, H_o Criterion, Singular Systems, Unknown Inputs, Variable Time Delay

\section{INTRODUCTION}

Singular systems have been widely considered in the last decade (Dai, 1989; Darouach, 1995; Ezzine, 2010; Khadhraoui, 2014). Since standard state space are not applicable for such system description, singulars models have been developed for such purpose. Therefore, to generate the state vector we have to design an observer. When an unknown disturbance input is acting on such system, we have to estimate such input especially when we aim to handle fault detection or system control. Even there are some works that dealt with the design of an observer for linear singular systems in presence of additional unknown input (Darouach, 1996; Ezzine, 2011; Khadhraoui, 2014; Johnson, 1975), no attempt has been made for the estimation of such input.

Moreover, the use of sensors and actuators introduce time delay on the system input and state. Therefore, the designed observer should take into account the delays effect. Despite that the simplest approach would consist in replacing them by some finite-dimensional approximations (Fattouh, 1999; Darouach, 2005; Ezzine, 2011; Khadhraoui, 2014), constant delay is not the general method 
of modeling delay. Also, in last decade, many researches (Koeing, 2006; Souley, 2006; Khadhraoui, 2016) have been interested in the design of an observer for system with Variable Time Delay.

Filters for singular systems with additional unknown inputs and constant time delay have been widely considered in the last decade and several design methods have been proposed (Ezzine, 2011; Khadhraoui, 2016). However, only few researches (Darouach, 2005; Khadhraoui, 2016) have been proposed when the delay is variable.

Motivated by these facts, we propose a functional $H_{\infty}$ filter for singular unknown inputs system with the presence of variable time delay in state and known input vector. Moreover, it doesn't require a prior knowledge on the disturbance except its bounds.

Since the dynamics of the estimation error contains the derivative of the bounded disturbance a transformation has been made so that the structure of this dynamics becomes a singular system. This feature enables to find the optimum gain of the filter by resolution of Linear Matrix Inequalities (LMI) so that we estimate a functional of the state vector and a part of the considered unknown input.

The outlines of the paper are as follows. Section 2 presents the difference between current work and recent published work. The third section gives the problem to be solved is formulated. Section 4 presents the contribution of the paper by giving the design procedure for the functional filter. Using the unbiasedness conditions, the problem is transformed into a singular problem. A LMI approach is then applied to optimize the gain implemented in the filter. Section 5 summarizes the design algorithm presentation. Numerical example is presented in section 6 and the last section concludes the paper.

\section{RELATED WORK}

This paper presents a new design of functional $H_{\infty}$ filter for linear singular system with unknown variable time delay. Comparing with recent references (Ezzine, 2011; Khadhraoui, 2014, 2016) also (Koeing, 2006) our approach offers two contributions; Firstly, we estimate a functional state vector by reducing mathematical conditions and then reduce time calculation. Secondly the proposed $H_{\infty}$ filter of the unknown inputs systems, with unknown variable time delay can estimate also a part of the unknown inputs vectors. These contributions are of major importance for control purposes and faults detection.

\section{PROBLEM FORMULATION}

Consider the following continuous-time linear singular delayed system described by:

$$
\begin{aligned}
& E \dot{x}(t)=A x(t)+A_{d} x(t-\tau(t))+B u(t)+B_{d} u(t-\tau(t))+F_{1} v(t)+G_{1} w(t) \\
& y(t)=C x(t)+D u(t)+F_{2} v(t)+G_{2} w(t)
\end{aligned}
$$

where $x \in \mathbb{R}^{n}, u \in \mathbb{R}^{m}$ and $y \in \mathbb{R}^{p}$ are the state, the known input and the output vectors respectively. $v \in \mathbb{R}^{q}$ and $w \in \mathbb{R}^{k}$ summarises respectively all the components of the unknown input and of the bounded disturbances that act on dynamic and output. E, $A, A_{d}, B, B_{d}, F_{1}, F_{2}, G_{1}, G_{2}, D$ and $C$ are known matrices of appropriate dimensions.

For simplicity and without loss of generality, we assume that the variable delays on state and input are identical. This delay satisfies: 
$0 \leq \tau(t) \leq \tau_{m}$

with $\tau_{m}$ the upper bound of $\tau(t)$.

In the sequel we suppose that (Zasadzinski, 1998):

$$
\begin{aligned}
& \operatorname{rank}(E)=\bar{n}<n \\
& \operatorname{rank}\left[\begin{array}{l}
F_{1} \\
F_{2}
\end{array}\right]=q \\
& \operatorname{rank}\left[\begin{array}{l}
G_{1} \\
G_{2}
\end{array}\right]=k
\end{aligned}
$$

Then, there exist an orthogonal matrix $R \in \mathbb{R}^{p \times p}$ and a non singular matrix $R \in \mathbb{R}^{q \times q}$, such that:

$$
R^{T} F_{2} S=\left(\begin{array}{ll}
I_{\bar{q}} & 0_{12} \\
0_{21} & 0_{22}
\end{array}\right)
$$

with $0_{12} \in \mathbb{R}^{\bar{q} \times(q-\bar{q})}, 0_{21} \in \mathbb{R}^{(p-\bar{q}) \times \bar{q}}$ and $0_{22} \in \mathbb{R}^{(p-\bar{q}) \times(q-\bar{q})}$ such that $0_{x y}$ is a matrix the component of which are all equal to zero.

By multiplying (1.b) by $R^{T}$ we obtain:

$$
\begin{aligned}
& y_{1}(t)=C_{1} x(t)+D_{1} u(t)+v_{1}(t)+G_{21} w(t) \\
& y_{2}(t)=C_{2} x(t)+D_{2} u(t)+G_{22} w(t)
\end{aligned}
$$

where:

$$
\begin{aligned}
& R^{T} y(t)=\left[\begin{array}{l}
y_{1} \\
y_{2}
\end{array}\right] \text { with } y_{1} \in \mathbb{R}^{\bar{q}} \text { and } y_{2} \in \mathbb{R}^{p-\bar{q}} \\
& R^{T} C=\left[\begin{array}{l}
C_{1} \\
C_{2}
\end{array}\right] \text { with } C_{1} \in \mathbb{R}^{\bar{q} \times n} \text { and } C_{2} \in \mathbb{R}^{(p-\bar{q}) \times n} \\
& R^{T} D=\left[\begin{array}{l}
D_{1} \\
D_{2}
\end{array}\right] \text { with } D_{1} \in \mathbb{R}^{\bar{q} \times m} \text { and } D_{2} \in \mathbb{R}^{(p-\bar{q}) \times m}
\end{aligned}
$$


and:

$R^{T} G_{2}=\left[\begin{array}{l}G_{21} \\ G_{22}\end{array}\right]$ with $G_{21} \in \mathbb{R}^{\bar{q} \times k}$ and $G_{22} \in \mathbb{R}^{(p-\bar{q}) \times k}$

for:

$R^{T} F_{2} S S^{-1}=\left(\begin{array}{ll}I_{\bar{q}} & 0_{12} \\ 0_{21} & 0_{22}\end{array}\right) S^{-1}$

Similarly, Equation (1a) becomes:

$E \dot{x}(t)=\bar{A} x(t)+A_{d} x(t-\tau(t))+\bar{B} u(t)+B_{d} u(t-\tau(t))+F_{11} y_{1}(t)+F_{12} v_{2}(t)+\bar{G} w(t)$

where:

$F_{1} S=\left[\begin{array}{ll}F_{11} & F_{12}\end{array}\right], \bar{A}=A-F_{11} C_{1}, \bar{B}=B-F_{11} D_{1}, \bar{G}=G_{1}-F_{11} G_{21}$

with $\bar{A} \in \mathbb{R}^{n \times n}, \bar{B} \in \mathbb{R}^{n \times m}, \bar{G} \in \mathbb{R}^{n \times k}, F_{11} \in \mathbb{R}^{n \times \bar{q}}$ and $F_{12} \in \mathbb{R}^{n \times(q-\bar{q})}$ and writing:

$S^{-1} v(t)=\left[\begin{array}{l}v_{1}(t) \\ v_{2}(t)\end{array}\right]$ with $v_{1}(t) \in \mathbb{R}^{\bar{q}}$ and $v_{2}(t) \in \mathbb{R}^{q-\bar{q}}$

So:

$R^{T} F_{2} v(t)=\left(\begin{array}{cc}I_{\bar{q}} & 0_{12} \\ 0_{21} & 0_{22}\end{array}\right)\left(\begin{array}{l}v_{1}(t) \\ v_{2}(t)\end{array}\right)=\left(\begin{array}{c}v_{1}(t) \\ 0_{q-\bar{q}}\end{array}\right)$

Then system (1) becomes:

$$
\begin{aligned}
& E \dot{x}(t)=\bar{A} x(t)+A_{d} x(t-\tau(t))+\bar{B} u(t)+B_{d} u(t-\tau(t))+F_{11} y_{1}(t)+F_{12} v_{2}(t)+\bar{G} w(t) \\
& y_{1}(t)=C_{1} x(t)+D_{1} u(t)+v_{1}(t)+G_{21} w(t) \\
& y_{2}(t)=C_{2} x(t)+D_{2} u(t)+G_{22} w(t)
\end{aligned}
$$




\subsection{Objective}

We aim to design a functional filter for linear singular delayed system. The considered system is affected by a bounded variable time delay acting on the state and the known input vectors. The functional filter estimates a part of the unknown input $v_{1}(t)$ and a linear combination of the state vector components $x_{i}, i=1 \ldots n$, using only the input and output measurements. This filter is defined by:

$$
z(t)=\left(\begin{array}{c}
L x(t) \\
v_{1}(t)
\end{array}\right) \in \mathbb{R}^{r+\bar{q}}
$$

with $L \in \mathbb{R}^{r \times n}$.

By replacing $v_{1}(t)$ by its expression derived from (11b), $z(t)$ can be written as:

$$
z(t)=\bar{L} x(t)+\bar{D}_{1} u(t)+\bar{G}_{21} w(t)+\bar{I} y_{1}(t)
$$

with:

$$
\bar{L}=\left[\begin{array}{c}
L \\
-C_{1}
\end{array}\right], \bar{D}_{1}=\left[\begin{array}{c}
0_{r \times m} \\
-D_{1}
\end{array}\right], \bar{G}_{21}=\left[\begin{array}{c}
0_{r \times k} \\
-G_{21}
\end{array}\right], \bar{I}=\left[\begin{array}{c}
0_{r \times \bar{q}} \\
\bar{I}_{\bar{q}}
\end{array}\right]
$$

with $\bar{L} \in \mathbb{R}^{(r+\bar{q}) \times n}, \operatorname{rank}(\bar{L})=r+\bar{q}<n$.

The functional $H_{\infty}$ filter estimate $z(t)$ described by Equation (13) use only the system known input $u(t)$ and output $y(t)$ as shown in Figure 1.

Figure 1. The functional $H_{\infty}$ filter design

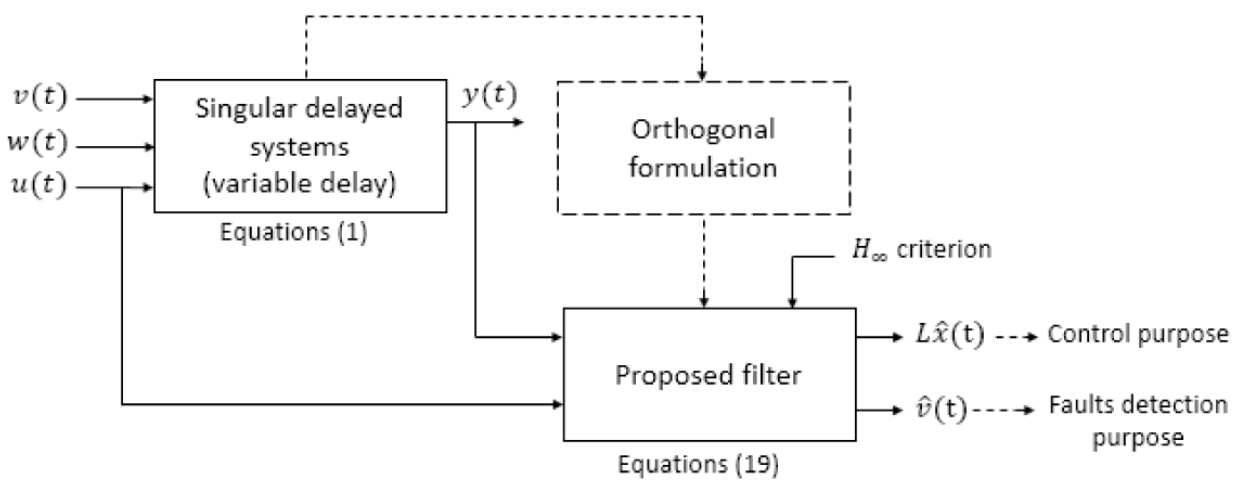


In the sequel of the paper we suppose that (Darouach, 1995):

$\operatorname{rank}\left(\begin{array}{c}E \\ C_{2} \\ \bar{L}\end{array}\right)=\operatorname{rank}\left(\begin{array}{c}E \\ C_{2}\end{array}\right)$

Then there exists a non singular matrix $T$ :

$T=\left(\begin{array}{ll}a & b \\ c & d\end{array}\right)$

such as:

$a E+b C_{2}=\bar{L}$

$c E+d C_{2}=0_{(p-\bar{q}) \times n}$

with $a \in \mathbb{R}^{(r+\bar{q}) \times n}, b \in \mathbb{R}^{(r+\bar{q}) \times(p-\bar{q})}, c \in \mathbb{R}^{(p-\bar{q}) \times n}$ and $d \in \mathbb{R}^{(p-\bar{q}) \times(p-\bar{q})}$.

\section{TIME DOMAIN DESIGN}

The purpose of the paper is to design a functional filter of the system defined by (11) and (13) of the form:

$$
\begin{aligned}
& \dot{\eta}(t)=M \eta(t)+M_{d} \eta(t-\tau(t))+K u(t)+K_{d} u(t-\tau(t)) \\
& \hat{z}(t)=\eta(t)+P u(t)+H_{1} y_{1}(t)+H_{2} y_{2}(t)
\end{aligned}
$$

where $\eta(t) \in \mathbb{R}^{r+\bar{q}}$ is the state vector of the filter and $\hat{z}(t)$ is the estimate of the functional state $\bar{z}(t) \in \mathbb{R}^{r+\bar{q}}$. The matrices $M, M_{d}, K, K_{d}, N_{1}, N_{2}, N_{d}, P, H_{1}$ and $H_{2}$ will be determined using the LMI approach.

\subsection{Conditions of the Functional Filter Synthesis}

Let's define $e(t)$ as the time estimation error:

$e(t)=z(t)-\hat{z}(t)$

Using (13) and (19b), e(t) is given by: 
$e(t)=\left(\bar{L}-H_{2} C_{2}\right) x(t)+\left(\bar{D}_{1}-P-H_{2} D_{2}\right) u(t)+\left(\bar{G}_{21}-H_{2} G_{22}\right) w(t)$

We assume that:

$P=\bar{D}_{1}-H_{2} D_{2}$

$H_{1}=\bar{I}$

So the Equation (21) is equivalent to:

$e(t)=\left(\bar{L}-H_{2} C_{2}\right) x(t)+\left(\bar{G}_{21}-H_{2} G_{22}\right) w(t)-\eta(t)$

Using (17) and (18) we can write:

$$
\begin{aligned}
& e(t)=\left(a E+b C_{2}-H_{2} C_{2}\right) x(t)+\beta\left(c E+d C_{2}\right) x(t)+\left(\bar{G}_{21}-H_{2} G_{22}\right) w(t)-\eta(t) \\
& =(a+\beta c) E x(t)+\left(b+\beta d-H_{2}\right) C_{2} x(t)+\left(\bar{G}_{21}-H_{2} G_{22}\right) w(t)-\eta(t)
\end{aligned}
$$

where $\beta \in \mathbb{R}^{(r+\bar{q}) \times(p-\bar{q})}$.

We assume that:

$$
H_{2}=b+\beta d
$$

So Equation (25) can be written in the following form:

$e(t)=\varphi \operatorname{Ex}(t)-\eta(t)+\left(\bar{G}_{21}-H_{2} G_{22}\right) w(t)$

with:

$\varphi=a+\beta c, \varphi \in \mathbb{R}^{(r+\bar{q}) \times n}$

The problem of the functional filter is to determine $M, M_{d}, K, K_{d}, N_{1}, N_{2}, N_{d}, P, H_{1}$ and $H_{2}$ such that:

$\lim _{t \rightarrow \infty} e(t)=0$, if $w=0$

The filtering error (27) is asymptotically stable and satisfy the $H_{\infty}$ performance. 
The $H_{\infty}$ criterion is given by:

$0<H_{\epsilon w \infty}=\sup _{w \neq 0} \frac{\epsilon_{2}}{w_{2}}<\gamma$

with $H_{\epsilon w}(s)=\frac{\epsilon(s)}{w(s)}$ is a transfer matrix and $\gamma$ is a positive scalar.

For this, we propose the following theorem.

\subsubsection{Theorem 1}

The unbiasedness estimation error given by (27) relative to system (11), (13) and functional $H_{\infty}$ filter (19) is verified such that:

$\dot{e}(t)=M e(t)+M_{d} e(t-\tau(t))+\alpha w(t)+\varsigma w(t-\tau(t))+\delta \dot{w}(t)$

with:

$$
\begin{aligned}
& \alpha=\varphi \bar{G}-M\left(\bar{G}_{21}-H_{2} G_{22}\right) N_{2} G_{22} \\
& \varsigma=-M_{d}\left(\bar{G}_{21}-H_{2} G_{22}\right) N_{d} G_{22} \\
& \delta=\bar{G}_{21}-H_{2} G_{22}
\end{aligned}
$$

$\alpha, \varsigma$ and $\delta \in \mathbb{R}^{(r+\bar{q}) \times k}$ if and only if the following Equations are satisfied:

$$
\begin{aligned}
& \varphi \bar{A}-N_{2} C_{2}-M \varphi E=0_{(r+\bar{q}) \times n} \\
& \varphi A_{d}-N_{d} C_{2}-M_{d} \varphi E=0_{(r+\bar{q}) \times n} \\
& \varphi \bar{B}-N_{2} D_{2}=K \\
& \varphi B_{d}-N_{d} D_{2}=K_{d} \\
& \varphi F_{11}=N_{1} \\
& \varphi F_{12}=0_{(r+\bar{q}) \times(p-\bar{q})}
\end{aligned}
$$

\subsubsection{Proof 1}

The time derivative of $e(t)$ given in (27) is: 


$$
\dot{e}(t)=\varphi E \dot{x}(t)-\dot{\eta}(t)+\left(\bar{G}_{21}-H_{2} G_{22}\right) \dot{w}(t)
$$

Replacing $E \dot{x}(t)$ and $\dot{\eta}(t)$ by their expressions given by (11a) and (19a) respectively, we have:

$$
\begin{aligned}
& \dot{e}(t)=M e(t)+M_{d} e(t-\tau(t))+\left[\varphi \bar{G}-M\left(\bar{G}_{21}-H_{2} G_{22}\right)-N_{2} G_{22}\right] w(t) \\
& +\left[\bar{G}_{21}-H_{2} G_{22}\right] \dot{w}(t)+\left[-M_{d}\left(\bar{G}_{21}-H_{2} G_{22}\right)-N_{d} G_{22}\right] w(t-\tau(t)) \\
& +\left[\varphi \bar{A}-N_{2} C_{2}-M \varphi E\right] x(t)+\left[\varphi A_{d}-N_{d} C_{2}-M_{d} \varphi E\right] x(t-\tau(t)) \\
& +\left[\varphi \bar{B}-N_{2} D_{2}-K\right] u(t)+\left[\varphi B_{d}-N_{d} D_{2}-K_{d}\right] u(t-\tau(t)) \\
& +\left[\varphi F_{11}-N_{1}\right] y_{1}(t)+\varphi F_{12} v_{2}(t)
\end{aligned}
$$

Using conditions(i)-(vi)oftheorem 1, andassuming that $w(t)=0, \dot{e}(t)=M e(t)+M_{d} e(t-\tau(t))$. Therefore $\lim _{t \rightarrow \infty} e(t)=0$.

\subsection{Determination of Observer Matrices}

At this stage, we propose to design the functional filter dependently from the state delay using a Lyapunov-Krasovskii stability theory (Krasovskii, 1963) and a LMI approach. So, the estimated functional state $\hat{z}(t)$ converges asymptotically to the real one $z(t)$ with the satisfaction of condition (29).

Now, replacing $\varphi$ given by its expression (28) in conditions i), ii) and vi) of theorem 1 and using (18), we have:By replacing (23) in conditions i) and iv) of theorem 1 and using (13), we have:

$$
\begin{aligned}
& a \bar{A}=M a E+J C_{2}-\beta c \bar{A} \\
& a A_{d}=M_{d} a E+J_{d} C_{2}-\beta c A_{d}
\end{aligned}
$$

and:

$$
a F_{12}=-\beta c F_{12}
$$

with:

$$
J=N_{2}-M \beta d
$$

$$
J_{d}=N_{d}-M_{d} \beta d
$$

Equations (36) - (38) can be written in the following matrix form: 
$\Gamma=\Omega \Theta$

with:

$\Omega=\left[\begin{array}{lllll}M & M_{d} & J & J_{d} & \beta\end{array}\right]$

$\Theta=\left(\begin{array}{ccc} & = & = \\ a E & 0_{1} & 0_{2} \\ = & & = \\ 0_{1} & a E & 0_{2} \\ & = & = \\ C_{2} & 0_{3} & 0_{4} \\ = & & = \\ 0_{3} & C_{2} & 0_{4} \\ -c \bar{A} & -c A_{d} & -c F_{12}\end{array}\right)$

$\Gamma=\left[\begin{array}{lll}a \bar{A} & a A_{d} & a F_{12}\end{array}\right]$

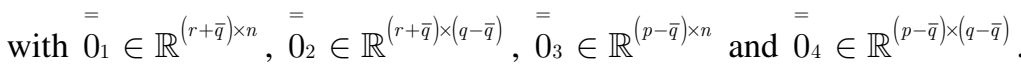

We note that the general Solution (41) exists if and only if (Zasadzinski, 1998):

$\operatorname{rank}\left[\begin{array}{l}\Theta \\ \Gamma\end{array}\right]=\operatorname{rank}(\Theta)$

Therefore, by respecting the condition (36):

$\Omega=\Gamma \Theta^{+}-Z\left(\bar{I}-\Theta \Theta^{+}\right)$

where $\bar{I}$ is an identity matrix of dimension $(2 r+3 p-\bar{q}), \Theta^{+}$is the generalized inverse of matrix $\Theta$ and $Z \in \mathbb{R}^{(r+\bar{q}) \times(2 r+3 p-\bar{q})}$ is an arbitrary matrix, that will be determined later using the LMI approach. The unknown matrix $M$ is defined by:

$M=\Omega\left(\begin{array}{c}I_{r+\bar{q}} \\ 0_{1} \\ 0_{2} \\ 0_{2} \\ 0_{2}\end{array}\right)=M_{1}+Z M_{2}$ 
$0_{1} \in \mathbb{R}^{(r+\bar{q}) \times(r+\bar{q})}$ and $0_{2} \in \mathbb{R}^{(r+\bar{q}) \times(p-\bar{q})}$

By replacing $\Omega$ by its expression given by (46) in (47), we obtain:

$$
M_{1}=\Gamma \Theta^{+}\left(\begin{array}{c}
I_{r+\bar{q}} \\
0_{1} \\
0_{2} \\
0_{2} \\
0_{2}
\end{array}\right), M_{2}\left(\bar{I}-\Theta \Theta^{+}\right)\left(\begin{array}{c}
I_{r+\bar{q}} \\
0_{1} \\
0_{2} \\
0_{2} \\
0_{2}
\end{array}\right)
$$

The unknown matrix $M_{d}$ is defined by:

$$
M=\Omega\left(\begin{array}{c}
0_{1} \\
I_{r+\bar{q}} \\
0_{2} \\
0_{2} \\
0_{2}
\end{array}\right)=M_{d 1}+Z M_{d 2}
$$

with:

$$
M_{d 1}=\Gamma \Theta^{+}\left(\begin{array}{c}
0_{1} \\
I_{r+\bar{q}} \\
0_{2} \\
0_{2} \\
0_{2}
\end{array}\right), M_{2}\left(I-\Theta \Theta^{+}\right)\left(\begin{array}{c}
0_{1} \\
I_{r+\bar{q}} \\
0_{2} \\
0_{2} \\
0_{2}
\end{array}\right)
$$

Similarly, $J$ is obtained such:

$$
\begin{aligned}
& J=\Omega\left(\begin{array}{c}
0_{1} \\
0_{1} \\
\bar{I}_{1} \\
0_{2} \\
0_{2}
\end{array}\right),=J_{1}+Z J_{2} \\
& \bar{I}_{1} \in \mathbb{R}^{(p-\bar{q}) \times(r+\bar{q})}
\end{aligned}
$$

with: 
$J_{1}=\Gamma \Theta^{+}\left(\begin{array}{l}0_{1} \\ 0_{1} \\ \bar{I}_{1} \\ 0_{2} \\ 0_{2}\end{array}\right), M_{2}\left(=-\Theta-\Theta \Theta^{+}\right)\left(\begin{array}{l}0_{1} \\ 0_{1} \\ \bar{I}_{1} \\ 0_{2} \\ 0_{2}\end{array}\right)$

$J_{d}$ is obtained such:

$J_{d}=\Omega\left(\begin{array}{c}0_{1} \\ 0_{1} \\ 0_{2} \\ \bar{I}_{1} \\ 0_{2}\end{array}\right),=J_{d 1}+Z J_{d 2}$

with:

$J_{d 1}=\Gamma \Theta^{+}\left(\begin{array}{l}0_{1} \\ 0_{1} \\ 0_{2} \\ \bar{I}_{1} \\ 0_{2}\end{array}\right), M_{2}\left(=-\Theta \Theta^{+}\right)\left(\begin{array}{l}0_{1} \\ 0_{1} \\ 0_{2} \\ \bar{I}_{1} \\ 0_{2}\end{array}\right)$

and $\beta$ is obtained such:

$\beta=\Omega\left(\begin{array}{l}0_{1} \\ 0_{1} \\ 0_{2} \\ 0_{2} \\ \bar{I}_{1}\end{array}\right)=\beta_{1}+Z \beta_{2}$

with:

$$
\beta_{1}=\Gamma \Theta^{+}\left(\begin{array}{l}
0_{1} \\
0_{1} \\
0_{2} \\
0_{2} \\
\bar{I}_{1}
\end{array}\right), \beta_{2}\left(=-\Theta \Theta^{+}\right)\left(\begin{array}{l}
0_{1} \\
0_{1} \\
0_{2} \\
0_{2} \\
\bar{I}_{1}
\end{array}\right)
$$


Using (39), (47) and (55) we can write $N_{2} G_{22}$ such that:

$$
\begin{aligned}
& N_{2} G_{22}=(J+M \beta d) G_{22} \\
& =J G_{22}+\left(M_{1}+Z M_{2}\right)\left(\beta_{1}+Z \beta_{2}\right) d G_{22} \\
& =J G_{22}+M_{1}\left(\beta_{1}+Z \beta_{2}\right) d G_{22}+\left(Z M_{2} \beta_{1}+Z M_{2} Z \beta_{2}\right) d G_{22}
\end{aligned}
$$

Similarly using (40), (49) and (55) $N_{d} G_{22}$ can be written as:

$$
\begin{aligned}
& N_{d} G_{22}=\left(J_{d}+M_{d} \beta d\right) G_{22} \\
& =J_{d} G_{22}+\left(M_{d 1}+Z M_{d 2}\right)\left(\beta_{1}+Z \beta_{2}\right) d G_{22} \\
& =J_{d} G_{22}+M_{d 1}\left(\beta_{1}+Z \beta_{2}\right) d G_{22}+\left(Z M_{d 2} \beta_{1}+Z M_{d 2} Z \beta_{2}\right) d G_{22}
\end{aligned}
$$

The matrices $N_{2} G_{22}$ and $N_{d} G_{22}$ have a bi-linearity $\left(Z M_{2} Z\right)$ and $\left(Z M_{d 2} Z\right)$. In order to avoid this bi-linearity we assume that the gain matrix $Z$ satisfies the following relation:

$$
Z \beta_{2}=0_{(r+\bar{q}) \times(p-\bar{q})}
$$

So there always exists a matrix $Z_{1} \in \mathbb{R}^{(r+\bar{q}) \times(2 r+3 p-\bar{q})}$ such that:

$$
Z=Z_{1}\left(\bar{I}-\beta_{2} \beta_{2}^{+}\right)=Z_{1} \Delta
$$

At this stage we avoid the gain $Z$ and we consider the new gain $Z_{1}$. Then $M, M_{d}, J, J_{d}$ and $\beta$ are defined as:

$$
\begin{aligned}
& M=M_{1}+Z_{1} \Delta M_{2} \\
& M_{d}=M_{d 1}+Z_{1} \Delta M_{d 2} \\
& J=J_{1}+Z_{1} \Delta J_{2} \\
& J_{d}=J_{d 1}+Z_{1} \Delta J_{d 2}
\end{aligned}
$$


$\beta=\beta_{1}$

Rewriting (31) - (33) and using (26) and (61) - (64) we can deduct that:

$\alpha=\alpha_{1}+Z_{1} \Delta \alpha_{2}$

$\varsigma=\varsigma_{1}+Z_{1} \Delta \varsigma_{2}$

$\delta=\bar{G}_{21}-b G_{22}-\beta_{1} d G_{22}$

with:

$\alpha_{1}=\varphi \bar{G}-M_{1}\left(\bar{G}_{21}-b G_{22}\right)-J_{1} G_{22}$

$\alpha_{2}=-M_{2}\left(\bar{G}_{21}-b G_{22}\right)-J_{2} G_{22}$

$\varsigma_{1}=-M_{d 1}\left(\bar{G}_{21}-b G_{22}\right)-J_{d 1} G_{22}$

$\varsigma_{2}=-M_{d 2}\left(\bar{G}_{21}-b G_{22}\right)-J_{d 2} G_{22}$

Hence all filter matrices are determined if and only if the matrix $Z_{1}$ is known which will be proposed in the following.

The error dynamics given by (30) contains the time derivative of $\dot{w}(t)$. This problem is generally solved by adding an additional constraint on the filter matrices or by choosing a new type of norm for the system. Based on (Khadhraoui, 2014) we propose to rewrite (30) into a singular form. Then the singular Equation is given:

$\left[\begin{array}{cc}I_{r+\bar{q}} & -\delta \\ \overline{0}_{21} & \overline{0}_{22}\end{array}\right]\left[\begin{array}{c}\dot{e}(t) \\ \dot{w}(t)\end{array}\right]=\left[\begin{array}{cc}M & \alpha \\ \overline{0}_{21} & -I_{k}\end{array}\right]\left[\begin{array}{c}e(t) \\ w(t)\end{array}\right]+\left[\begin{array}{cc}M_{d} & \delta \\ \overline{0}_{21} & \overline{0}_{22}\end{array}\right]\left[\begin{array}{c}e(t-\tau(t)) \\ w(t-\tau(t))\end{array}\right]+\left[\begin{array}{c}\overline{0}_{21}^{T} \\ I_{k}\end{array}\right] w(t)$

with $\overline{0}_{21} \in \mathbb{R}^{k \times(r+\bar{q})}$ and $\overline{0}_{22} \in \mathbb{R}^{k \times k}$.

Let's consider that: 
$l=r+\bar{q}+k, \epsilon(t)=\left[\begin{array}{c}e(t) \\ w(t)\end{array}\right] \in \mathbb{R}^{l}$

Then we have:

$\rho \epsilon^{\cdot}(t)=\tilde{M} \epsilon(t)+\tilde{M}_{d} \epsilon(t-\tau(t))+\tilde{K} w(t)$

where:

$\rho=\left[\begin{array}{ll}I_{r+\bar{q}} & -\delta \\ \overline{0}_{21} & \overline{0}_{22}\end{array}\right]$

$\tilde{M}=\left[\begin{array}{cc}M & \alpha \\ \overline{0}_{21} & -I_{k}\end{array}\right]$

and:

$\tilde{M}_{d}=\left[\begin{array}{ll}M_{d} & \delta \\ \overline{0}_{21} & \overline{0}_{22}\end{array}\right]$

$\tilde{K}=\left[\begin{array}{c}\overline{0}_{21}^{T} \\ I_{k}\end{array}\right]$

with $\rho, \tilde{M}, \tilde{M}_{d} \in \mathbb{R}^{l \times l}$ and $\tilde{K} \in \mathbb{R}^{l \times k}$.

A LMI approach is proposed in the following theorem to determine the gain $Z_{1}$ which parametrizes the $H_{\infty}$ filter matrices.

\subsubsection{Theorem 2}

System (19) is a functional filter for system (1) if there exist matrices $P_{1}=P_{1}^{T}>0, P_{2}, P_{3}=P_{3}^{T}>0$ and $Y$ solution of the following LMI that holds for a given $\gamma>0$ :

$\left(\begin{array}{ll}\psi & X \\ * & \Pi\end{array}\right)<0$

and:

$\tilde{P} \rho=\rho^{T} \tilde{P}^{T}>0$ 


$$
\tilde{P}=\left(\begin{array}{cc}
P_{1} & P_{2}^{T} \\
P_{2} & \mathrm{P}_{3}
\end{array}\right)
$$

$P_{2}=-\delta^{T} P_{1}$

where $\delta$ and $\rho$ are given by (68) and (76) and the matrices $\psi, X$ and $\Pi$ are given in the Appendix. The gain $Z_{1}$ is given by:

$$
Z_{1}=P_{1}^{-1} Y
$$

\subsubsection{Proof 2}

To prove the Theorem 2 we consider the following Lyapunov-Krasovskii functional:

$V(t)=V_{1}(t)+V_{2}(t)+V_{3}(t)$

with:

$$
\begin{aligned}
& V_{1}(t)=\epsilon(t)^{T} \tilde{P} \rho \epsilon(t) \\
& V_{2}(t)=\int_{0}^{\tau(t)} \int_{t-\theta}^{t} \epsilon(\mu)^{T} \tilde{M}^{T} \tilde{P} \tilde{M} \epsilon(\mu) d \mu d \theta \\
& V_{3}(t)=\int_{0}^{\tau(t)} \int_{t-\theta}^{t} \epsilon(\mu)^{T} \tilde{M}_{d}^{T} \widetilde{P}_{d} \epsilon(\mu) d \mu d \theta
\end{aligned}
$$

where $\tilde{P} \in \mathbb{R}^{l \times l}$ is symmetric positive and definite matrix and $\epsilon(t)$ is defined in (74).

According to condition (2), $V(t) \geq 0, \forall t \in \mathbb{R}^{+}$and knowing that $\epsilon(\mu)^{T} \tilde{M}^{T} \widetilde{P} \tilde{M} \epsilon(\mu)$ and $\epsilon(\mu)^{T} \tilde{M}_{d}^{T} \widetilde{P} \tilde{M}_{d} \epsilon(\mu)$ are positive scalars, we can write:

$0 \leq V(t) \leq \bar{V}(t)$

with: 
$\bar{V}(t)=V_{1}(t)+\tau_{m} V_{21}(t)+\tau_{m} V_{31}(t)$

$V_{21}(t)=\int_{0}^{\tau_{m}} \int_{t-\theta}^{t} \epsilon(\mu)^{T} \tilde{M}^{T} \widetilde{P} \tilde{M} \epsilon(\mu) d \mu d \theta$

$V_{31}(t)=\int_{0}^{\tau_{m}} \int_{t-\theta}^{t} \epsilon(\mu)^{T} \tilde{M}_{d}^{T} \widetilde{P} \tilde{M}_{d} \epsilon(\mu) d \mu d \theta$

Based on (89), if $\lim _{t \rightarrow \infty} \bar{V}(t)=0$ then $\lim _{t \rightarrow \infty} V(t)=0$.

To prove that $\bar{V}(t) \approx 0$ when $t \rightarrow \infty$ we can prove that $\bar{V}(t) \approx 0$ when $t \rightarrow \infty$ (see (Khadhraoui, 2014)).

The time derivative of the functional $\bar{V}(t)$ is:

$\dot{V}(t)=\dot{V}_{1}(t)+\tau_{m} \dot{V}_{21}(t)+\tau_{m} \dot{V}_{31}(t)$

where:

$\dot{V}_{1}(t)=\epsilon^{\cdot}(t)^{T} \rho^{T} \tilde{P} \epsilon(t)+\epsilon(t)^{T} \tilde{P} \rho \dot{\epsilon}(t)$

Using Equation (75), we deduct that:

$$
\begin{aligned}
& \dot{V}_{1}(t)=\epsilon(t)^{T}\left[\tilde{M}^{T} \tilde{P}+\tilde{P} \tilde{M}\right] \epsilon(t)+\epsilon(t)^{T} \tilde{P} \tilde{K} w(t)+\epsilon(t)^{T} \tilde{P} \tilde{M}_{d} \epsilon(t-\tau(t)) \\
& +w(t)^{T} \tilde{K}^{T} \tilde{P} \epsilon(t)+\epsilon(t-\tau(t))^{T} \tilde{M}_{d}^{T} \tilde{P} \epsilon(t)
\end{aligned}
$$

Moreover:

$$
\dot{V}_{21}(t)=\tau_{m} \epsilon(t)^{T} \tilde{M}^{T} \tilde{P} \tilde{M} \epsilon(t)-\int_{0}^{\tau_{m}} \epsilon(t-\theta)^{T} \tilde{M}^{T} \tilde{P} \tilde{M} \epsilon(t-\theta) d \theta
$$

and:

$$
\dot{V}_{31}(t)=\tau_{m} \epsilon(t)^{T} \tilde{M}_{d}^{T} \tilde{P} \tilde{M}_{d} \epsilon(t)-\int_{0}^{\tau_{m}} \epsilon(t-\theta)^{T} \tilde{M}_{d}^{T} \widetilde{P}_{M_{d}} \epsilon(t-\theta) d \theta
$$


Let's define that:

$\xi(t-\theta)=\tilde{M} \epsilon(t-\theta)$

and:

$\xi_{d}(t-\theta)=\tilde{M}_{d} \epsilon(t-\theta)$

with $\xi$ and $\xi_{d} \in \mathbb{R}^{l}$.

So we can write:

$\dot{V}_{21}(t)=\tau_{m} \epsilon(t)^{T} \tilde{M}^{T} \tilde{P} \tilde{M} \epsilon(t)-\int_{0}^{\tau_{m}} \xi(t-\theta)^{T} \widetilde{P} \xi(t-\theta) d \theta$

and:

$\dot{V}_{31}(t)=\tau_{m} \epsilon(t)^{T} \tilde{M}_{d}^{T} \tilde{P} \tilde{M}_{d} \epsilon(t)-\int_{0}^{\tau_{m}} \xi_{d}(t-\theta)^{T} \widetilde{P} \xi_{d}(t-\theta) d \theta$

As $\theta$ is finite, the quantities $\xi(t-\theta)$ and $\xi_{d}(t-\theta)$, given by (98) and (99) respectively, satisfy:

$\lim _{t \rightarrow \infty} \xi(t-\theta)=\lim _{t \rightarrow \infty} \xi(t)$

and:

$\lim _{t \rightarrow \infty} \xi_{d}(t-\theta)=\lim _{t \rightarrow \infty} \xi_{d}(t)$

Therefore:

$\lim _{t \rightarrow \infty} \int_{0}^{\tau_{m}} \xi(t-\theta)^{T} \widetilde{P} \xi(t-\theta) d \theta=\tau_{m} \lim _{t \rightarrow \infty} \xi(t)^{T} \widetilde{P} \xi(t)$

and:

$\lim _{t \rightarrow \infty} \int_{0}^{\tau_{m}} \xi_{d}(t-\theta)^{T} \widetilde{P} \xi_{d}(t-\theta) d \theta=\tau_{m} \lim _{t \rightarrow \infty} \xi_{d}(t)^{T} \stackrel{\widetilde{P}}{\sim} \xi_{d}(t)$

Then we suppose that: 
$\lambda=\lim _{t \rightarrow \infty} \xi(t)$

$v=\lim _{t \rightarrow \infty} \xi_{d}(t)$

$\varepsilon=\lim _{t \rightarrow \infty} \epsilon(t)$

Uniform asymptotic stability implies that:

$\lim _{t \rightarrow \infty} \frac{\dot{U}}{V}(t) \leq 0$

According to (93)-(108) the Equation (93) is equivalent to:

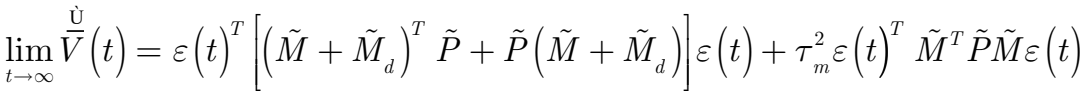

$$
\begin{aligned}
& +\tau_{m}^{2} \varepsilon(t)^{T} \tilde{M}_{d}^{T} \tilde{P} \tilde{M}_{d} \varepsilon(t)+w(t)^{T} \tilde{K}^{T} \tilde{P} \varepsilon(t)+\varepsilon(t)^{T} \tilde{P} \tilde{K} w(t)-\tau_{m}^{2} \lambda^{T} \tilde{P} \lambda-\tau_{m}^{2} v^{T} \tilde{P} v
\end{aligned}
$$

In order to establish sufficient conditions for existence of (19) according to $H_{\infty}$ criterion, we should verify the following inequality (Khadhraoui, 2016):

$$
H(\varepsilon, t)=\dot{V}(t)+\varepsilon(t)^{T} \varepsilon(t)-\gamma^{2} w(t)^{T} w(t)<0
$$

which can be written as follows:

$$
\left[\begin{array}{llll}
\varepsilon^{T} & \lambda^{T} & v^{T} & w^{T}
\end{array}\right] \Psi\left[\begin{array}{c}
\varepsilon \\
\lambda \\
v \\
w
\end{array}\right]
$$

with:

$$
\Psi=\left(\begin{array}{cccc}
Q & 0_{l \times l} & 0_{l \times l} & \tilde{P} \tilde{K} \\
* & -\tau_{m}^{2} \tilde{P} & 0_{l \times l} & 0_{l \times k} \\
* & * & -\tau_{m}^{2} \tilde{P} & 0_{l \times k} \\
* & * & * & -\gamma^{2} I_{k}
\end{array}\right)
$$


and:

$Q=\left(\tilde{M}+\tilde{M}_{d}\right)^{T} \tilde{P}+\tilde{P}\left(\tilde{M}+\tilde{M}_{d}\right)+\tau_{m}^{2} \tilde{M}^{T} \tilde{P} \tilde{M}+\tau_{m}^{2} \tilde{M}_{d}^{T} \tilde{P} \tilde{M}_{d}+I_{l}$

To avoid the quadratic form present in Equation (113), we propose to rewrite this Equation as following form:

$$
\Psi=U-\Phi^{T} \Xi^{-1} \Phi
$$

with:

$\Phi=\left(\begin{array}{cccc}\tau_{m} \tilde{M} & 0_{l \times l} & 0_{l \times l} & 0_{l \times k} \\ 0_{l \times l} & I_{l} & 0_{l \times l} & 0_{l \times k} \\ \tau_{m} \tilde{M}_{d} & 0_{l \times l} & I_{l} & 0_{l \times k} \\ 0_{k \times l} & 0_{k \times l} & 0_{k \times l} & \gamma I_{k}\end{array}\right)$

$\Xi=-\left(\begin{array}{cccc}\tilde{P}^{-1} & 0_{l \times l} & 0_{l \times l} & 0_{l \times k} \\ * & \tilde{P}^{-1} & 0_{l \times l} & 0_{l \times k} \\ * & * & \tilde{P}^{-1} & 0_{l \times k} \\ * & * & * & I_{k}\end{array}\right)$

and:

$U=\left(\begin{array}{cccc}Q_{1} & 0_{l \times l} & -\tau_{m} \tilde{M}_{d}^{T} \tilde{P} & \tilde{P} \tilde{K} \\ * & -\left(\tau_{m}^{2}+1\right) \tilde{P} & 0_{l \times l} & 0_{l \times k} \\ * & * & -\left(\tau_{m}^{2}+1\right) \tilde{P} & 0_{l \times k} \\ * & * & * & -2 \gamma^{2} I_{k}\end{array}\right)$

$Q_{1}=\left(\tilde{M}+\tilde{M}_{d}\right)^{T} \tilde{P}+\tilde{P}\left(\tilde{M}+\tilde{M}_{d}\right)+I_{l}$

According to the Schur lemma, $\Psi<0$ and $\Xi<0$ if and only if:

$$
\Lambda=\left[\begin{array}{cc}
\Xi & \Phi \\
\Phi^{T} & U
\end{array}\right]<0
$$

Now we propose to apply a congruence transformation (Khadhraoui, 2014) to $\Lambda$ such as: 
where $\Sigma$ is a non singular matrix given by:

$$
\sum=\left(\begin{array}{cccccccc}
\widetilde{P} & 0_{l \times l} & 0_{l \times l} & 0_{l \times k} & 0_{l \times l} & 0_{l \times l} & 0_{l \times l} & 0_{l \times k} \\
* & \widetilde{P} & 0_{l \times l} & 0_{l \times k} & 0_{l \times l} & 0_{l \times l} & 0_{l \times l} & 0_{l \times k} \\
* & * & \widetilde{P} & 0_{l \times k} & 0_{l \times l} & 0_{l \times l} & 0_{l \times l} & 0_{l \times k} \\
* & * & * & I_{k} & 0_{k \times l} & 0_{k \times l} & 0_{k \times l} & 0_{k \times k} \\
* & * & * & * & I_{l} & 0_{l \times l} & 0_{l \times l} & 0_{l \times k} \\
* & * & * & * & * & I_{l} & 0_{l \times l} & 0_{l \times k} \\
* & * & * & * & * & * & I_{l} & 0_{l \times k} \\
* & * & * & * & * & * & * & \widetilde{P}
\end{array}\right)
$$

Then by replacing $\tilde{M}, \tilde{M}_{d}$ and $\tilde{K}$ by their expressions given by (77), (78) and (79) respectively in (56), theorem 2 holds.

\section{ALGORITHM FOR FUNCTIONAL FILTER DESIGN}

Step 1: Check that conditions 1), 2), 3) and 4) are satisfied.

Step 2: Compute matrices $R$ and $\mathrm{S}$ using (3).

Step 3: Check that condition (15) is verified.

Step 4: Compute matrix $T$ using (17) and (18).

Step 5: Compute $\Theta$ and $\Gamma$ using (43) and (44).

Step 6: Deduce the values of matrices $M_{1}, M_{2}, M_{d 1}, M_{d 2}, J_{1}, J_{2}, J_{d 1}, J_{d 2}, \beta_{1}$ and $\beta_{2}$ from (47)-(56).

Step 7: Verify that the condition (45) is satisfied. Then resolution of the proposed LMI (80), gives the gain matrix $Z_{1}$.

Step 8: Compute $M, M_{d}, J, J_{d}$ and $\beta$ using (61)-(65).

Step 9: Get matrices $P, H_{1}, H_{2}, N_{2}$ and $N_{d}$ using (22), (23), (26), (39) and (40).

Step 10: Get matrices $K, K_{d}$ and $N_{1}$ using iii) - v) from theorem 1.

\section{NUMERICAL EXAMPLES}

Let's consider system (1) where:

$$
\begin{aligned}
& E=\left(\begin{array}{cc}
1 & 0 \\
-1 & 0
\end{array}\right), A=\left(\begin{array}{ll}
1 & -1 \\
2 & -1
\end{array}\right), A_{d}=\left(\begin{array}{ll}
1 & 0 \\
0 & 1
\end{array}\right), B=\left(\begin{array}{l}
1 \\
0
\end{array}\right), B_{d}=\left(\begin{array}{l}
0 \\
1
\end{array}\right), D=\left(\begin{array}{l}
1 \\
1
\end{array}\right) \\
& F_{1}=\left(\begin{array}{ll}
1 & 0 \\
0 & 1
\end{array}\right), F_{2}=\left(\begin{array}{ll}
0 & 0 \\
1 & 2
\end{array}\right), C=\left(\begin{array}{cc}
3 & 2 \\
1 & -1
\end{array}\right), G_{1}=\left(\begin{array}{c}
-1 \\
1
\end{array}\right) G_{2}=\left(\begin{array}{l}
1 \\
0
\end{array}\right), L=\left(\begin{array}{ll}
1 & -1
\end{array}\right)
\end{aligned}
$$


The variable state and known input delay is a sinusoid such:

$\tau(t)=-0.5 \sin (1.2 t)+1$

According to (2), we have $0.5 \leq \tau(t) \leq 1.5, \forall t \in \mathbb{R}^{+}$.

The $H_{\infty}$ criterion is bounded by $\gamma=0,7$.

We suppose that $u(t), w(t)$ and $u(t)$ are respectively given by Figures 2-4.

Figure 2. Input signal $u(t)$

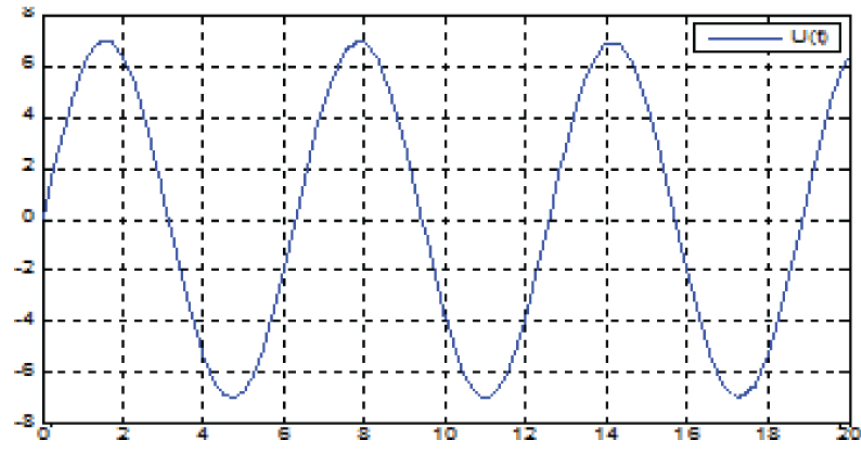

Figure 3. Bounded disturbance $w(t)$

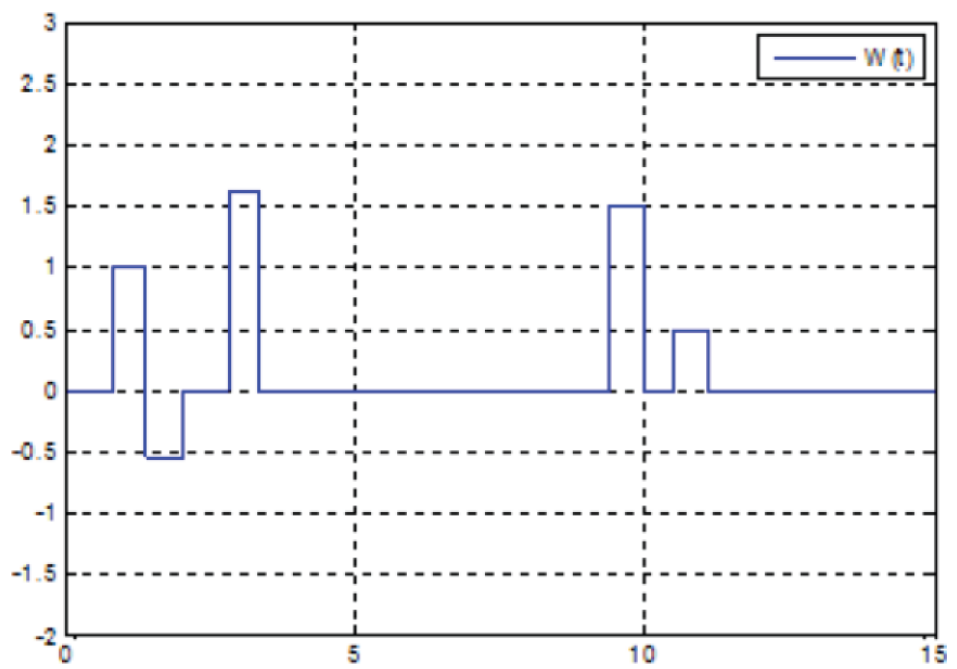


Figure 4. Unknown inputs $v(t)$
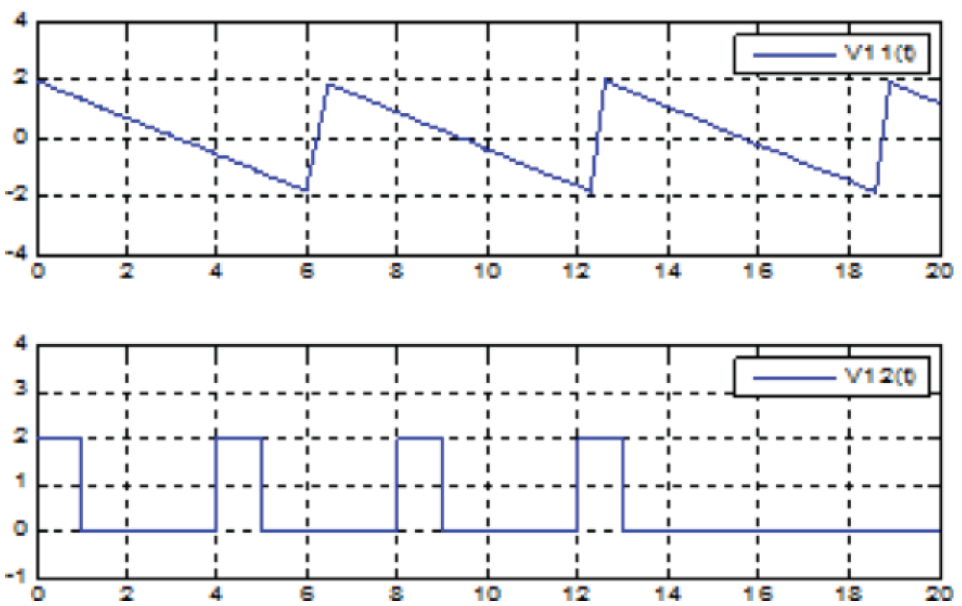

Following the procedure proposed below, we obtain:

$R=\left(\begin{array}{cc}0 & -1 \\ -1 & 0\end{array}\right), S=\left(\begin{array}{cc}-0.2 & -0.894 \\ -0.4 & 0.447\end{array}\right)$

By multiplying the unknown inputs $v(t)$ by $S^{-1}$ we have (Figure 5):

Figure 5. Part of unknown inputs $v_{1}(t)$

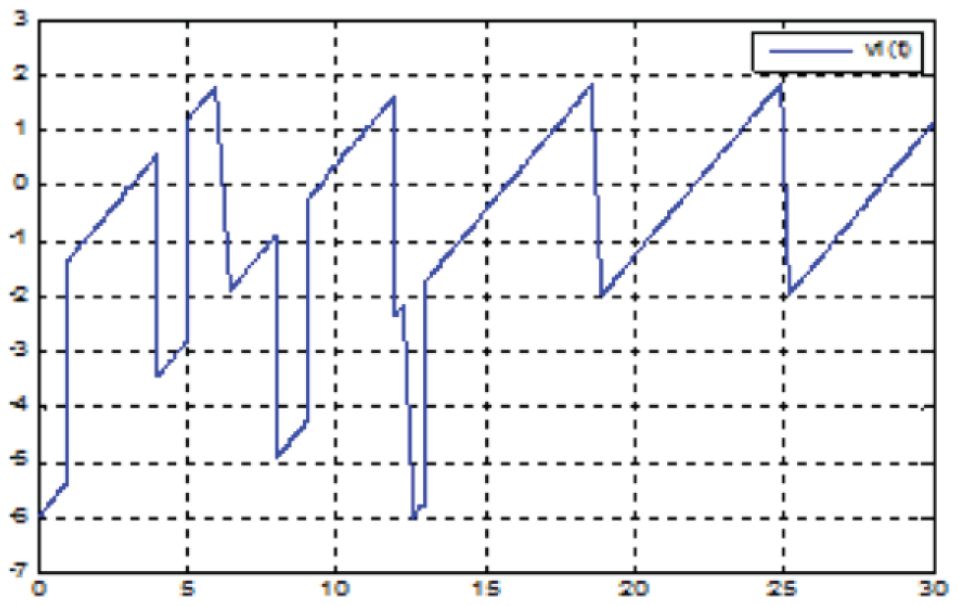


The resolution of LMI (80) gives:

$$
\begin{aligned}
& P_{1}=\left(\begin{array}{cc}
51.4 & 51.37 \\
51.37 & 51.4
\end{array}\right), P_{2}=\left(\begin{array}{llllll}
-0.01 & 0.01
\end{array}\right), P_{3}=0.708 \\
& Y=\left(\begin{array}{llllllll}
-20.79 & -20.79 & -14.97 & -14.97 & 0 & 0 & 0 \\
-20.79 & -20.79 & -14.97 & -14.97 & 0 & 0 & 0
\end{array}\right) \\
& Z_{1}=\left(\begin{array}{lllllll}
-0.2 & -0.2 & -0.14 & -0.14 & 0 & 0 & 0 \\
-0.2 & -0.2 & -0.14 & -0.14 & 0 & 0 & 0
\end{array}\right)
\end{aligned}
$$

So, the functional filter matrices values are given as follows:

$$
\begin{aligned}
& M=\left(\begin{array}{cc}
-5.20 & 4.79 \\
4.79 & -5.20
\end{array}\right), M_{d}=\left(\begin{array}{cc}
0.850 & -1.14 \\
-1.14 & 0.85
\end{array}\right), N_{1}=\left(\begin{array}{c}
0.5 \\
-0.5
\end{array}\right), N_{2}=\left(\begin{array}{c}
-1 \\
1
\end{array}\right), N_{d}=\left(\begin{array}{c}
0.5 \\
-0.5
\end{array}\right) \\
& K=\left(\begin{array}{c}
-1 \\
1
\end{array}\right), K_{d}=\left(\begin{array}{c}
-0.5 \\
0.5
\end{array}\right), H_{1}=\left(\begin{array}{l}
0 \\
1
\end{array}\right), H_{2}=\left(\begin{array}{c}
0.5 \\
-0.5
\end{array}\right), P=\left(\begin{array}{c}
0.5 \\
0.5
\end{array}\right)
\end{aligned}
$$

Next figures are carried out using the Simulink platform of Matlab. Figure 6 shows a comparison between the real and the estimated functional state vector.

Figure 6. Functional state $z(t)$

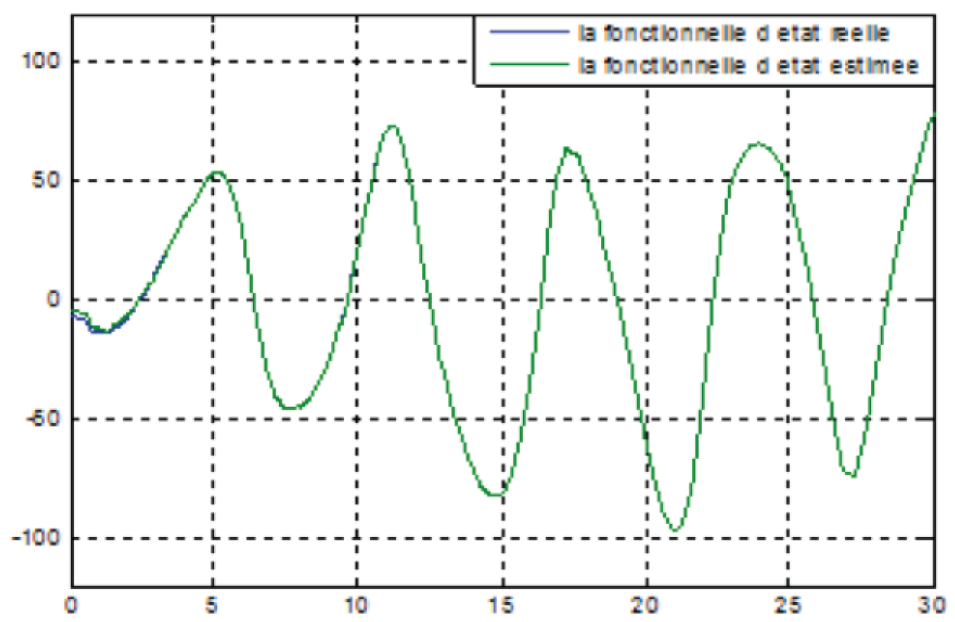

Figure 7 represents the real and the estimated values of part $v_{1}(t)$.

We can remark as it shown in Figure 6 and Figure 7 the time domain behavior of the functional $H_{\infty}$ filter, so it has a rapid convergence dynamic. The effect of the disturbance on the estimation error is evaluated by $H_{e w \infty}=0,52<\gamma$ 
Figure 7. Unknown inputs $v_{1}(t)$

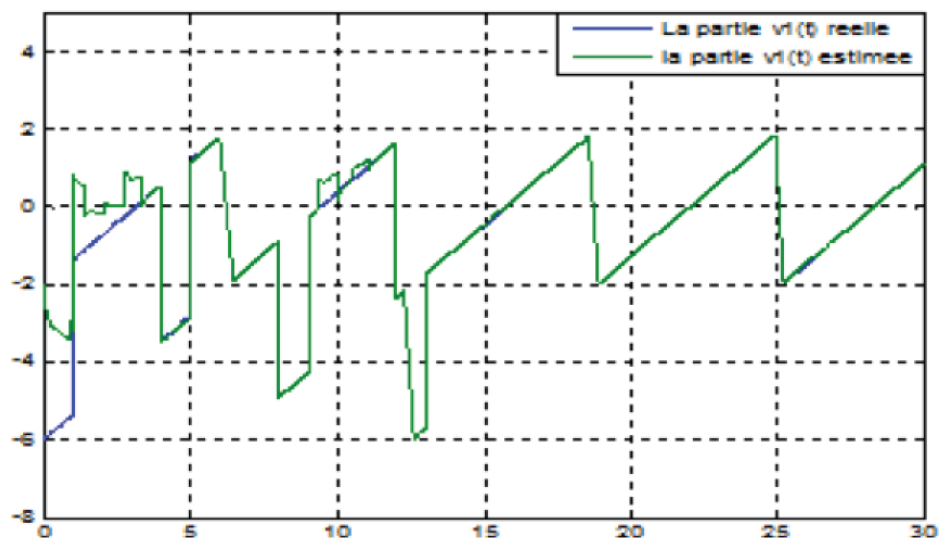

Then the estimation error of the functional state $z(t)$ and the unknown input $v_{1}(t)$ are given respectively by Figure 8 and Figure 9 .

We note that the error estimation achieves the permanent phase at $t=6 \mathrm{~s}$ with respect to the $H_{\infty}$ disturbance attenuation criterion. So, we highlight the effectiveness of the design techniques dependently on the delay with an application on a variable state delay.

Figure 8. Estimation error of functional state

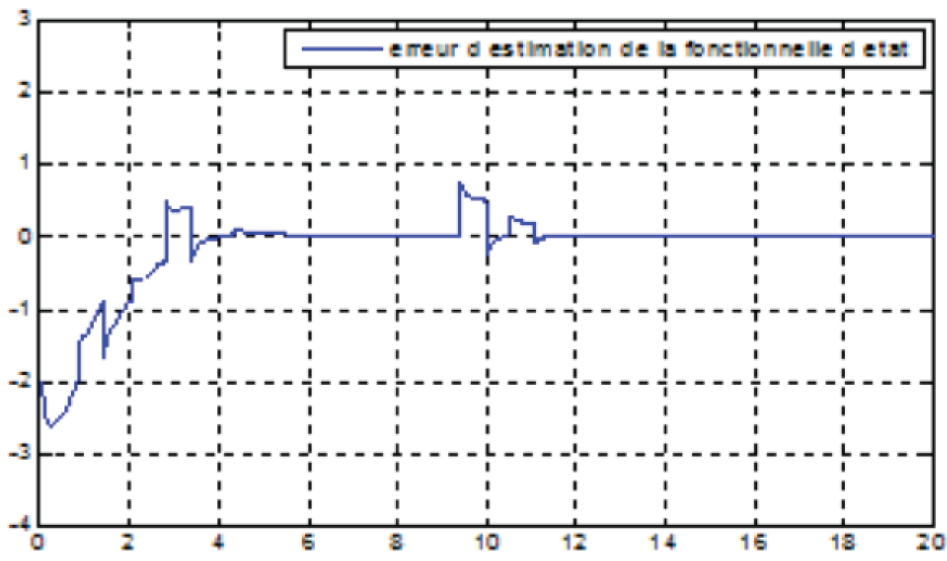

\section{CONCLUSION}

In this paper, we have proposed a new method for the design functional $H_{\infty}$ filter of linear singular systems with bounded disturbance, unknown inputs and bounded variable time delay acting on the state and the known input vectors. Using only the input and output measurements, the designed filter will estimate a reduced functional state and a part of the unknown inputs, which can be used on control purposes and handle fault detection. 
Figure 9. Estimation error of unknown input

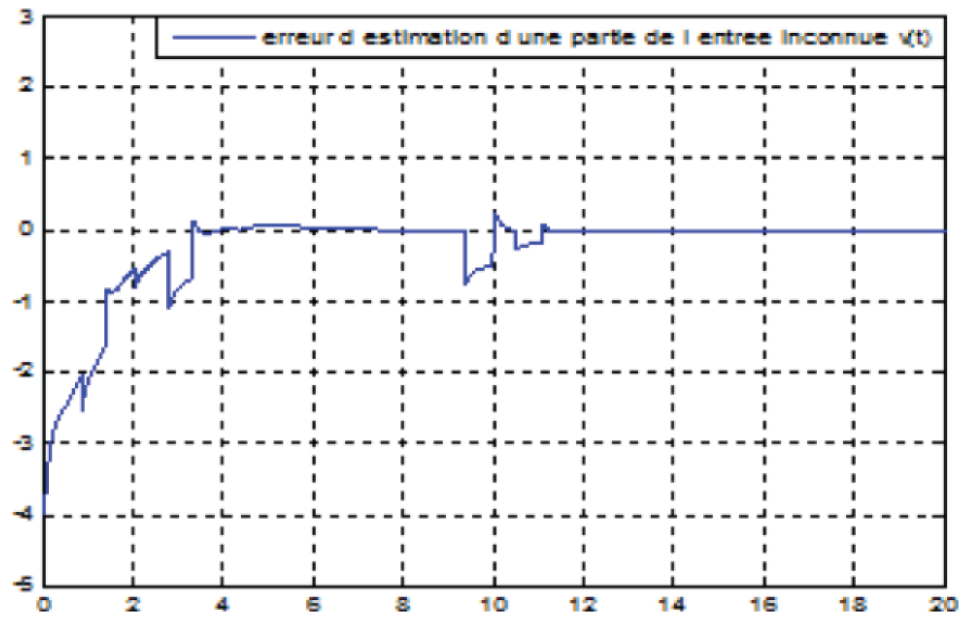

This procedure is based on the unbiasedness of the estimation error, the Lyapunov-Krasovskii stability theory and the disturbance minimizing effect on the estimation error.

Such results are of major of importance for fault detection and control using feedback state schema. 


\section{REFERENCES}

Balochian, S., \& Nahid, R. (2018). Fractional-Order Optimal Control of Fractional-Order Linear Vibration Systems with Time Delay. International Journal of System Dynamics Applications, 7(3), 72-93. doi:10.4018/ IJSDA.2018070104

Bouzaida, S., \& Sakly, A. (2018). Adaptive Neuro-Fuzzy Sliding Mode Controller. International Journal of System Dynamics Applications, 7(2), 34-54. doi:10.4018/IJSDA.2018040103

Dai, L. (1989). Singular Control Systems. In Lecture Notes in Control and Information Sciences (vol. 118). Springer-Verlag.

Darouach, M., \& Boutayeb, M. (1995). Design of observers for descriptor systems. IEEE Transactions on Automatic Control, 40(7), 1323-1327. doi:10.1109/9.400467

Darouach, M., Zasadzinski, M., \& Hayar, M. (1996). Reduced-Order Observer Design for Descriptor Systems with Unknown Inputs. IEEE Transactions on Automatic Control, 41(7), 1068-1072. doi:10.1109/9.508918

Darouach, M. (2005). Linear Functional Observers for systems with delays in state variables: The discrete time case. IEEE Transactions on Automatic Control, 50(2), 228-233. doi:10.1109/TAC.2004.841932

Ezzine, M., Darouach, M., Souley, H., \& Messaoud, H. (2010). Synthese temporelle et frequentielle de filtres d'ordre plein pour les systemes singuliers. Conference Internationale Francophone d'Automatique.

Ezzine, M., Darouach, M., Souley, H., \& Messaoud, H. (2011). Unknown Inputs Functional Observers Designs For Descriptor Systems with Constant Time Delay. IFAC, 44(1), 1162-1167.

Ezzine, M., Darouach, M., Souley, H., \& Messaoud, H. (2012). A controller design based on a functional $\mathrm{H}_{\infty}$. Automatica, 48(3), 542-549. doi:10.1016/j.automatica.2011.08.060

Fattouh, A., Sename, O., \& Dion, J.-M. (1999). Robust observer design for time-delay systems: A Riccati Equation approach. Kybernetika, 36(6), 753-764.

Fu, Y. M., Duan, G. R., \& Song, S. M. (2004). Design of unknown input observer for linear time-delay systems. International Journal of Control, Automation, and Systems, 2(4), 530-535.

Ghabi, J., Rhif, A., \& Vaidyanathan, S. (2018). Discrete Time Sliding Mode Control Scheme for Nonlinear Systems With Bounded Uncertainties. International Journal of System Dynamics Applications, 7(2), 15-33. doi:10.4018/IJSDA.2018040102

Hostetter, G., \& Meditch, J. (1973). Observing systems with unmeasurable inputs. IEEE Transactions on Automatic Control, 18(3), 307-308. doi:10.1109/TAC.1973.1100296

Hou, M., Zítek, P., \& Patton, R. J. (2002). An observer design for linear time-delay systems. IEEE Transactions on Automatic Control, 47(1), 121-125. doi:10.1109/9.981730

Johnson, C. (1975). On observers for systems with unknown and inaccessible inputs. International Journal of Control, 21(5), 825-831. doi:10.1080/00207177508922036

Khadhraoui, M., Ezzine, M., Messaoud, H., \& Darouch, M. (2014). Design of full order filter for delayed singular systems with unknown input and bounded disturbance. International Conference on Control, Decision and Information Technologies, 429-434.

Khadhraoui, M., Ezzine, M., \& Messaoud, H. (2014). Design of Full Order Observers with Unknown Inputs for Delayed Singular Systems with Constant Time Delay. International Conference on Control, Decision and Information Technologies, 423-428.

Khadhraoui, M., Ezzine, M., Messaoud, H., \& Darouch, M. (2014). Design of unknown input functional observers for delayed singular systems with state variable time delay. WSEAS Transactions on Systems and Control.

Khadhraoui, M., Ezzine, M., \& Messaoud, H. (2016). A controller design based on a functional $\mathrm{H}_{\infty}$ filter for delayed singular systems: The time and frequency domain cases. WSEAS Transactions on Systems, 48(3), 542-549. 
Koenig, D., \& Marx, B. (2004). Design of Observers for descriptor systems with delayed state and unknown inputs. American Control Conference, 5(5), 4806-4810. doi:10.23919/ACC.2004.1384073

Krasovskii, N. (1963). Stability of Motion. Stanford University Press.

Masubuchi, I., Kamitane, Y., Ohara, A., \& Suda, N. (1997). Ho control for descriptor systems: A matrix inequalities approach. Automatica, 33(4), 669-673. doi:10.1016/S0005-1098(96)00193-8

Meziane, S., Atarsia, L., \& Toufouti, R. (2018). A Global Stability of Linearizing Control of Induction Motor for PV Water Pumping Application. International Journal of System Dynamics Applications, 7(3), 31-56. doi:10.4018/IJSDA.2018070102

Niculescu, I. S. (2001). Delay effect on stability, A robust control approach. Springer Verlag.

Niculescu, S. I., Trofino-Neto, A., Dion, J. M., \& Dugard, L. (1995). Delay-dependent stability of linear systems with delayed state: An L.M.I. approach. Proc. of the 34th Conference on Decision and Control, 36(6), 1495-1496.

Niculescu, S. L., Dion, J. M., \& Dugard, L. (1998). Mixed Delay-Independent / Delay-Dependent Stability of Linear Systems With Delayed State: An L.M.I. Approach. IFAC, 31(9), 69-74. doi:10.1016/S14746670(17)41130-X

Seyed, H. N., \& Saeed, B. (2018). The Stability of a Class of Fractional Order Switching System with Time-Delay Actuator. International Journal of System Dynamics Applications, 7(1), 85-96. doi:10.4018/IJSDA.2018010105

Souley, H., Darouach, M., \& Zasadzinski, M. (2006). Approche LMI pour la synthèse des filtres $\mathrm{H}_{\infty}$ non Biaisés. Academic Press.

Verghese, G., Levy, B., \& Kailath, T. (1981). A generalized state-space for singular systems. IEEE Transactions on Automatic Control, 26(4), 811-831. doi:10.1109/TAC.1981.1102763

Zasadzinski, M., Rafaralahy, H., Mechmeche, C., \& Darouach, M. (1998). On Disturbance Decoupled Observers for a Class of Bilinear System. Journal of Dynamic Systems, Measurement, and Control, 120(3). 


\section{APPENDIX: MATRICES}

The matrices $\psi, X$ and $\Pi$ used in theorem 2 are defined as:

$$
\begin{aligned}
& \psi=\left(\begin{array}{cccc}
-\tilde{P} & 0_{l \times l} & 0_{l \times l} & 0_{l \times k} \\
* & -\tilde{P} & 0_{l \times l} & 0_{l \times k} \\
* & * & -\tilde{P} & 0_{l \times k} \\
* & * & * & -I_{k}
\end{array}\right) \\
& X=\left(\begin{array}{cccc}
\tau_{m} \tilde{P} \tilde{M} & 0_{l \times l} & 0_{l \times l} & 0_{l \times k} \\
0_{l \times l} & \tilde{P} & 0_{l \times l} & 0_{l \times k} \\
\tau_{m} \tilde{P} \tilde{M}_{d} & 0_{l \times l} & \tilde{P} & 0_{l \times k} \\
0_{k \times l} & 0_{k \times l} & 0_{k \times l} & \gamma I_{k}
\end{array}\right)
\end{aligned}
$$

Replacing $\tilde{M}, \tilde{M}_{d}$ and $\tilde{P}$ by their values in (77), (78) and (82) respectively, the matrix $X$ can be written as:

$$
X=\left(\begin{array}{lllllll}
X_{11} & X_{12} & X_{13} & X_{14} & X_{15} & X_{16} & X_{17} \\
X_{21} & X_{22} & X_{23} & X_{24} & X_{25} & X_{26} & X_{27} \\
X_{31} & X_{32} & X_{33} & X_{34} & X_{35} & X_{36} & X_{37} \\
X_{41} & X_{42} & X_{43} & X_{44} & X_{45} & X_{46} & X_{47} \\
X_{51} & X_{52} & X_{53} & X_{54} & X_{55} & X_{56} & X_{57} \\
X_{61} & X_{62} & X_{63} & X_{64} & X_{65} & X_{66} & X_{67} \\
X_{71} & X_{72} & X_{73} & X_{74} & X_{75} & X_{76} & X_{77}
\end{array}\right)
$$

where:

$$
\begin{aligned}
& X_{11}=\tau_{m} P_{1} M_{1}+\tau_{m} Y \Delta M_{2} \\
& X_{12}=\tau_{m} P_{1} \alpha_{1}+\tau_{m} Y \Delta \alpha_{2}+\tau_{m} P_{1} \delta \\
& X_{13}=X_{15}=X_{31}=X_{35}=X_{53}=0_{1} \\
& X_{14}=X_{16}=X_{17}=X_{32}=X_{36}=X_{37}=X_{54}=X_{57}=\overline{0}_{21}^{T} \\
& X_{21}=-\tau_{m} \delta^{T} P_{1} M_{1}-\tau_{m} \delta^{T} Y \Delta M_{2} \\
& X_{22}=-\tau_{m} \delta^{T} P_{1} \alpha_{1}-\tau_{m} \delta^{T} Y \Delta \alpha_{2}-\tau_{m} P_{3} \\
& X_{23}=X_{25}=X_{41}=X_{45}=X_{63}=X_{71}=X_{73}=X_{75}=\overline{0}_{21} \\
& X_{24}=X_{26}=X_{27}=X_{42}=X_{46}=X_{47}=X_{64}=X_{67}=X_{72}=X_{74}=X_{76}=0_{k \times k} \\
& X_{33}=X_{55}=p_{1} \\
& X_{34}=X_{56}=-P_{1} \delta
\end{aligned}
$$




$$
\begin{aligned}
& X_{43}=X_{65}=-\delta^{T} P_{1} \\
& X_{44}=X_{66}=P_{3} \\
& X_{51}=\tau_{m} P_{1} M_{d 1}+\tau_{m} Y \Delta M_{d 2} \\
& X_{52}=\tau_{m} P_{1} \varsigma_{1}+\tau_{m} Y^{\prime} \varsigma_{2} \\
& X_{61}=-\tau_{m} \delta^{T} P_{1} M_{d 1}-\tau_{m} \delta^{T} Y^{\prime} M_{d 2} \\
& X_{62}=-\tau_{m} \delta^{T} P_{1} \varsigma_{1}-\tau_{m} \delta^{T} Y^{\prime} \varsigma_{2} \\
& X_{77}=\gamma I_{k}
\end{aligned}
$$

and:

$$
\Pi=\left(\begin{array}{cccc}
Q_{1} & 0_{l \times l} & -\tau_{m} \tilde{M}_{d}^{T} \tilde{P} & \tilde{P} \tilde{K} \\
* & -\left(\tau_{m}^{2}+1\right) \tilde{P} & 0_{l \times l} & 0_{l \times k} \\
* & * & -\left(\tau_{m}^{2}+1\right) \tilde{P} & 0_{l \times k} \\
* & * & * & -2 \gamma^{2} I_{k}
\end{array}\right)
$$

Similarly by replacing $\tilde{M}_{d}, \tilde{K}, \tilde{P}$ and $Q_{1}$ by their values in (78), (79), (82) and (119) respectively, the matrix $\Pi$ can be written as:

$$
\begin{aligned}
& \Pi=\left(\begin{array}{ccccccc}
\Pi_{11} & \Pi_{12} & \Pi_{13} & \Pi_{14} & \Pi_{15} & \Pi_{16} & \Pi_{17} \\
* & \Pi_{22} & \Pi_{23} & \Pi_{24} & \Pi_{25} & \Pi_{26} & \Pi_{27} \\
* & * & \Pi_{33} & \Pi_{34} & \Pi_{35} & \Pi_{36} & \Pi_{37} \\
* & * & * & \Pi_{44} & \Pi_{45} & \Pi_{46} & \Pi_{47} \\
* & * & * & * & \Pi_{55} & \Pi_{56} & \Pi_{57} \\
* & * & * & * & * & \Pi_{66} & \Pi_{67} \\
* & * & * & * & * & * & \Pi_{77}
\end{array}\right) \\
& \Pi_{11}=M_{1}^{T} P_{1}+M_{2}^{T} \Delta^{T} Y^{T}+M_{d 1}^{T} P_{1}+M_{d 2}^{T} \Delta^{T} Y^{T}+P_{1} M_{1}+Y \Delta M_{2}+P_{1} M_{d 1}+Y \Delta M_{d 2}+I_{r+\bar{q}} \\
& \Pi_{12}=-M_{1}^{T} P_{1} \delta-M_{2}^{T} \Delta^{T} Y^{T} \delta-M_{d 1}^{T} P_{1} \delta+P_{1} \alpha_{1}+Y \Delta \alpha_{2}+P_{1} \varsigma_{1}+Y \Delta \varsigma_{2}+P_{1} \delta-M_{d 2}^{T} \Delta^{T} Y^{T} \delta \\
& \Pi_{13}=\Pi_{35}=0_{1} \\
& \Pi_{14}=\Pi_{36}=\Pi_{37}=\Pi_{57}=\overline{0}_{21}^{T} \\
& \Pi_{22}=-\alpha_{1}^{T} P_{1} \delta-\alpha_{2}^{T} \Delta^{T} Y^{T} \delta-\varsigma_{1}^{T} P_{1} \delta-\varsigma_{2}^{T} \Delta^{T} Y^{T} \delta-\delta^{T} P_{1} \alpha_{1} \\
& -\delta^{T} Y \Delta \alpha_{2}-\delta^{T} P_{1} \varsigma_{1}-\delta^{T} Y \Delta \varsigma_{2}-2 P_{3}+I_{k}
\end{aligned}
$$




$$
\begin{aligned}
& \Pi_{23}=\Pi_{45}=\overline{0}_{21} \\
& \Pi_{24}=\Pi_{46}=\Pi_{47}=\Pi_{67}=0_{k \times k} \\
& \Pi_{15}=-\tau_{m} M_{d 1}^{T} P_{1}-\tau_{m} M_{d 2}^{T} \Delta^{T} Y^{T} \\
& \Pi_{16}=\tau_{m} M_{d 1}^{T} P_{1} \delta+\tau_{m} M_{d 2}^{T} \Delta^{T} Y^{T} \delta \\
& \Pi_{25}=-\tau_{m} \varsigma_{1}^{T} P_{1}-\tau_{m} \varsigma_{2}^{T} \Delta^{T} Y^{T} \\
& \Pi_{26}=\tau_{m} \varsigma_{1}^{T} P_{1} \delta+\tau_{m} \varsigma_{2}^{T} \Delta^{T} Y^{T} \delta \\
& \Pi_{17}=-P_{1} \delta \\
& \Pi_{27}=P_{3} \\
& \Pi_{33}=\Pi_{55}=-\left(\tau_{m}^{2}+1\right) P_{1} \\
& \Pi_{34}=\Pi_{56}=\left(\tau_{m}^{2}+1\right) P_{1} \delta \\
& \Pi_{44}=\Pi_{66}=-\left(\tau_{m}^{2}+1\right) P_{3} \\
& \Pi_{77}=-2 \gamma^{2} I_{k}
\end{aligned}
$$

